\title{
Prosthetic Full Mouth Rehabilitation in a Case of Xerostomia following Radiation Therapy for Carcinoma of Esophagus: A Clinical Case Report
}

\author{
${ }^{1}$ Karunakar B Shetty, ${ }^{2}$ Deepa Jayashankar \\ ${ }^{1}$ Professor, Department of Prosthodontics, Bangalore Institute of Dental Sciences, Bengaluru, Karnataka, India \\ ${ }^{2}$ Ex-Postgraduate Student, Department of Prosthodontics, Bangalore Institute of Dental Sciences, Bengaluru, Karnataka, India
}

Correspondence: Karunakar B Shetty, Professor, Department of Prosthodontics, Bangalore Institute of Dental Sciences 5/3, Hosur Road, Bengaluru, Karnataka, India, Phone:0091-9902197761, Fax:0091-80-26563973, e-mail:dr_karans@hotmail.com

\section{ABSTRACT}

Aim of the study: Complete rehabilitation of a 38-year-old female patient with radiation caries and xerostomia postirradiation therapy for the treatment of carcinoma of esophagus, by a multidisciplinary approach involving specialties of periodontics, endodontics and prosthodontics.

Summary: This 38-year-old female patient presented with missing teeth, ill fitting prosthesis, radiation caries and xerostomia as a consequence of radiation therapy. In addition, the patient was severely psychologically affected not just due to the carcinoma, but also due to the adverse effects of cancer therapy. Therefore, rehabilitation of such a patient was aimed not just at careful oral reconstruction of lost and damaged structures but also towards providing a boost to her self-esteem. The outcome of this multidisciplinary approach led to a completely wellrehabilitated patient, both orally as well as psychologically.

Keywords: Cast partial denture, Endodontic treatment, Fixed partial prosthesis, Overdenture, Radiation caries, Xerostomia.

\section{INTRODUCTION}

The dental management of radiation induced caries and xerostomia is a complex process necessitating a multidisciplinary approach to successful management. It is the dentist's foremost responsibility to provide a complete solution to the compounding problem. A complete solution not only refers to the successful restoration of the dentition per se but also refers to the psychological and mental well being of the patient. The responsibility of complete oral rehabilitation lies not just with the dental specialties, but also necessitates periodic consultations with the concerned medical specialties. Thorough motivation of the patient to maintain the restored teeth is also an equally arduous task and forms an essential element of the treatment protocol. Although various management strategies have been suggested in the past,$^{1-3}$ the present case was handled keeping the best interests of the patient in mind and not offering any compromise as far as esthetics and function were concerned.

\section{CLINICAL CASE REPORT}

A 38-year-old female patient reported to department of prosthodontics, Bangalore Institute of Dental Sciences, Bengaluru, in March 2009 for the replacement of missing mandibular anterior teeth since last 2 months.

The patient had been diagnosed with carcinoma of the posterior cricoid region 4 years ago, for which chemotherapy and radiotherapy was administered and completed at Bangalore Institute of Oncology, Bengaluru, India, 3 years back. Since then, the patient has been visiting the institute at regular intervals for complete medical checkups. The patient was prescribed artificial saliva for the adverse effect of xerostomia associated with this form of therapy.

In January 2009, the patient complained of decay in the mandibular anterior teeth, and was diagnosed with radiation caries, probably a consequence of xerostomia. Since the teeth were not restorable, it was decided to extract these teeth in consultation with the oncologist and physician, who consented for the same, as there was no likelihood of development of any hazardous situation since the radiation and chemotherapy had been terminated 3 years ago. A few of the missing teeth had been replaced by removable partial dentures 2 years ago.

The patient was then referred to the department of prosthodontics a few weeks later for the replacement of missing teeth (Fig. 1A). She had a normal built, was physically healthy, but lacked confidence owing to her poor oral health status. The tongue appeared inflamed with depapillated areas and a white patch was seen on the dorsal surface, which was scrapable suggesting chronic atrophic candidiasis. Keratotic areas were also seen along the commissure of the lip suggestive of angular chelitis. In addition, the patient was on artificial saliva, i.e. carboxy methylcellulose which had been prescribed for the xerostomia. Generalized gingival inflammation with bleeding on probing was also reported. Root stumps of left maxillary central incisor, mandibular left canine and mandibular right 1st premolar were present. Improperly contoured acrylic crowns were present on teeth 11, 21, 22 and 23 (Fig. 1B). Faulty margins were also observed in the ceramic crowns on teeth 12, 13, 34 and 44. 
Removable partial dentures replacing maxillary and mandibular posterior teeth were also present.

Based on the above findings, the patient was asked to go in for the following investigations in order to formulate an appropriate treatment plan.

1. Intraoral periapical radiographs with respect to all teeth present.

2. An orthopantomogram.

3. Consent from the patient's physician/oncologist to carry out any kind of dental treatment.

In addition, the patient was advised to continue artificial saliva to keep the mouth well lubricated throughout the proposed treatment regime. Antifungal therapy (kenalog gel) for candidiasis and fluoride therapy for prevention of caries was also recommended.

The radiographic investigations showed crestal bone loss in all existing teeth. Tooth 11, 14, 21, 34 and 44 showed evidence of endodontic treatment. However, the patient complained of pain and sensitivity in these teeth.

Based on these reports, the following treatment plan was devised and implemented in coordination with the specialties of endodontics and periodontics.

The acrylic crowns in tooth 11, 21, 22 and 23 were first removed followed by the ceramic crowns in tooth $12,13,34$ and 44. The poor periodontal condition around these teeth necessitated the removal of these crowns, which was followed by complete oral prophylaxis and gingival curettage with the consent of the patient's physician. The patient was then placed on an appropriate maintenance protocol.

The previously endodontically treated teeth were further evaluated and it was decided that tooth 34 and 44 be retained with the intent of fabricating an overdenture following a repeat root canal therapy. Tooth length was determined with precision and without penetration of the periapical tissues. Instrumentation was such that a gradual recession from the foramen resulted as wider diameter instruments were used. This technique minimized periapical trauma and developed a solid apical stop to prevent overfilling and concomitant inflammation. Caustic irrigating solutions were avoided; sterile saline solution in copious amounts was used instead. ${ }^{4,5}$

A repeat endodontic treatment was also carried out in tooth 11,14 and 21. Intentional root canal therapy was carried out in tooth $12,22,23$. It was decided to retain these teeth with the objective of maintaining the natural esthetics of the maxillary anterior teeth. The posterior teeth were missing and retaining the anterior teeth would definitely aid in providing an esthetic advantage. Moreover, a combination approach of fixed and removable prostheses to rehabilitate partially edentulous patients is advantageous to xerostomic patients. This allows the removable partial denture design to be simplified and minimizes tissue coverage. ${ }^{6}$

On completion of the same, dowel core (cast metal) restorations were made on tooth 11 and 21 (Fig. 2A) followed by individual porcelain fused to metal crowns in tooth 11,21 ,
22 and 23 and a porcelain fused to metal fixed partial prosthesis with tooth 12, 13 and 14 (Fig. 3A). Cast core and dowel restorations may not be indicated in some cases because of caries susceptibility and the fact that some abutment root stumps lack sufficient retention. ${ }^{4}$ However, the root length in tooth 11 and 21 were adequate and there was sufficient retention available for the dowel core restorations in addition to the fact that artificial saliva and fluoride therapy was prescribed. In a dry oral environment, optimal reconstruction is with a nontissuebearing prosthesis. Fixed partial dentures should preferably have full-coverage retainers, and easily cleaned pontics and connectors. $^{6}$

A cast partial denture (CPD) for the missing upper posterior teeth was fabricated (Fig. 3B). Bilateral distal extension edentulous spaces (Kennedy’s class I) are by far best replaced by cast partial dentures. An appropriately designed prosthesis necessitates the placement of at least three positive rests on three sound abutments as widely apart as possible to aid in support, stability and retention and to provide adequate resistance to biomechanical forces. In keeping with this, cingulum rests and occlusal rests were used in the CPD design. ${ }^{7}$ The use of a lattice type of minor connector in the design was because it offers maximum stability. ${ }^{8}$ The borders of the anterior strap of the major connector should end in the valleys in the incisal rugae, making it less obtrusive. Moreover, the maxillary major connector should not cover the anterior rugae, 'the playground of the tongue' or the posterior soft palate. ${ }^{9}$ Therefore, the double strap anteroposterior major connector was designed which provides maximum rigidity for the least amount of metal bulk present. ${ }^{7}$ The I-bar direct retainer for the canine was designed, as it is more retentive and requires only minimal tooth coverage $\mathrm{e}^{10,11}$ and the circumferential clasp was preferred for the premolar as there were tissue undercuts, lack of vestibular depth, exostoses present and labially inclined abutment tooth. ${ }^{7}$ A retrospective study comparing the effectiveness of clasp designs, the distal rest and circumferential clasp system and I-bar system found that the success rate of either design is $74 \% .^{12}$ In addition to this, cingulum rests on canines were designed as indirect retainers because placing 1 or 2 additional rests on corresponding guiding plates, as far as possible from abutment rests helps distribution of forces, thereby improving support, stability and retention of the prosthesis. ${ }^{9}$

Lastly, cast metal copings with a chamfer finish line and dome preparation were done to create a postspace with tooth 34 and 44 (Fig. 2B) to receive an overdenture for the mandibular $\operatorname{arch}^{13,14}$ (Fig. 3B). Mirza and Dikshit, ${ }^{15}$ state that in patients who have received treatment doses of radiation to the head and neck, dentures may cause irritation and osteoradionecrosis, especially if the tissues are ischemic and atrophic. The soft tissue must be evaluated carefully before considering rehabilitation with a removable prosthesis. There are no objective data to determine when, if ever, a removable prosthesis may safely be inserted. Most authors recommend 1 year, but this can vary depending on tissue healing and patient history. ${ }^{15}$ Anticancer 

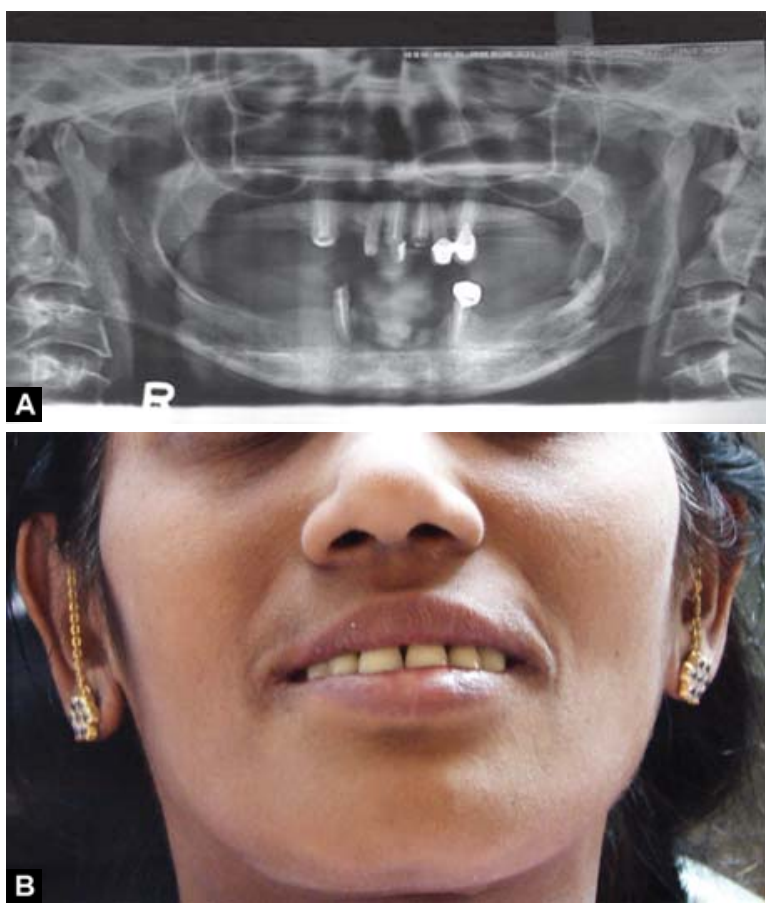

Figs 1A and B: (A) Preoperative orthopantomogram, (B) preoperative frontal profile view
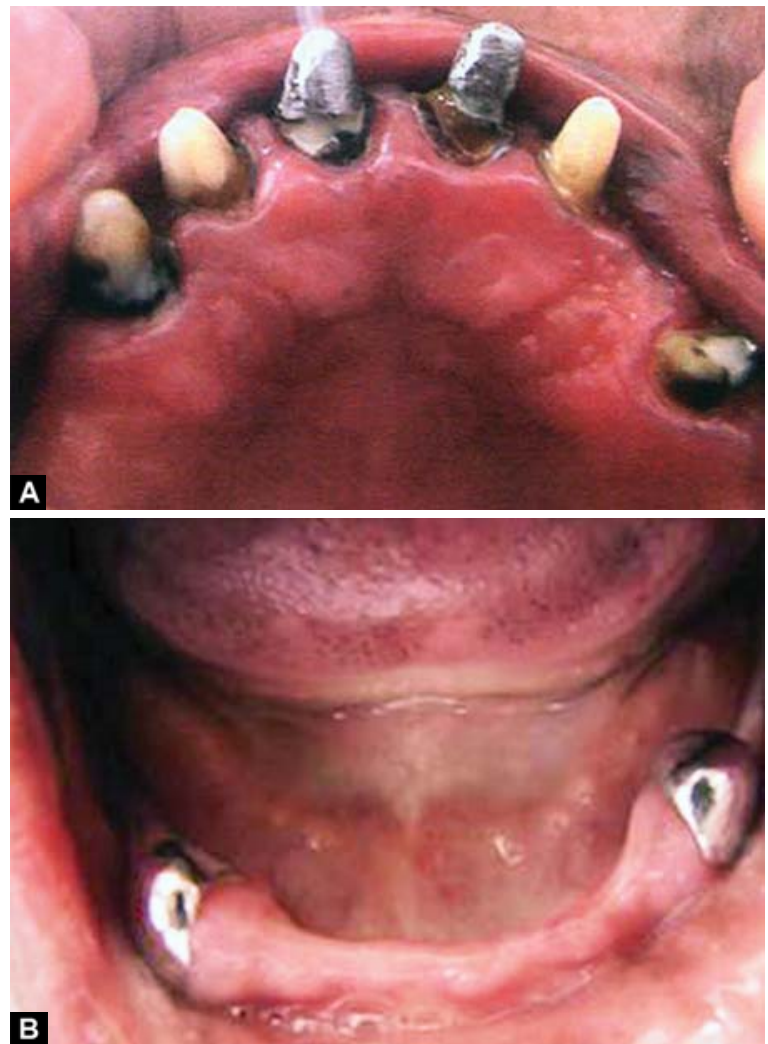

Figs 2A and B: (A) Dowel core restorations on tooth 11 and 21, endodontically treated tooth $12,14,22$ and 23 , (B) cast metal copings in tooth 34 and 44

therapy for the patient had already been completed 3 years ago. In addition, the patient had been regularly using the artificial saliva substitutes prescribed to her; hence, the tissue condition never posed any major problem.
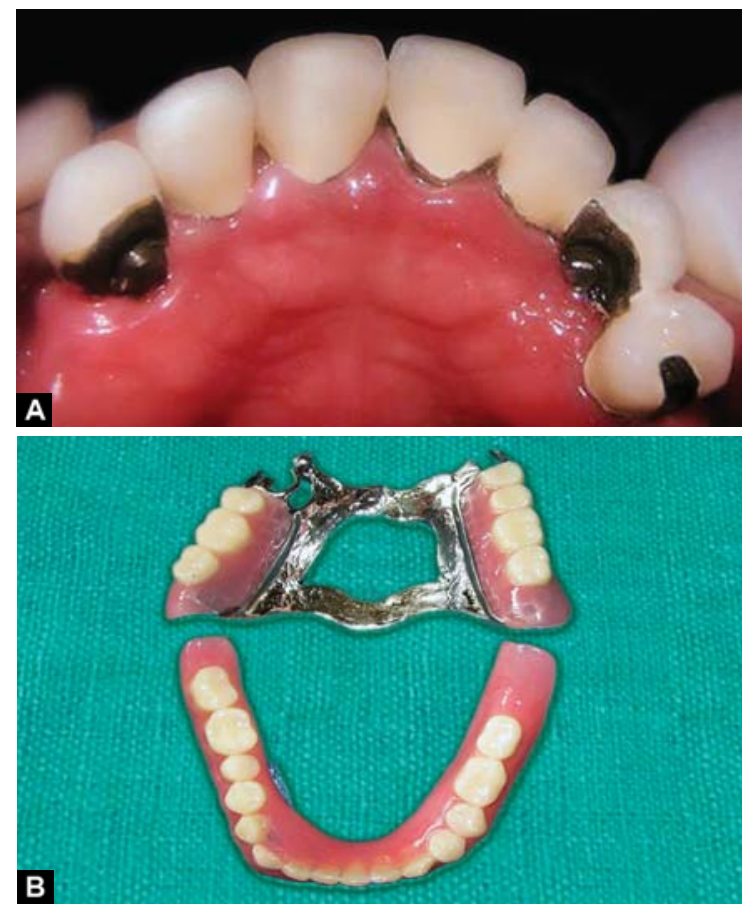

Figs $3 A$ and $B:(A)$ Porcelain fused to metal-fixed partial prosthesis with tooth 12,13 and 14 , porcelain fused to metal-individual crowns in tooth $11,21,22$ and 23, (B) maxillary cast partial denture, mandibular overdenture
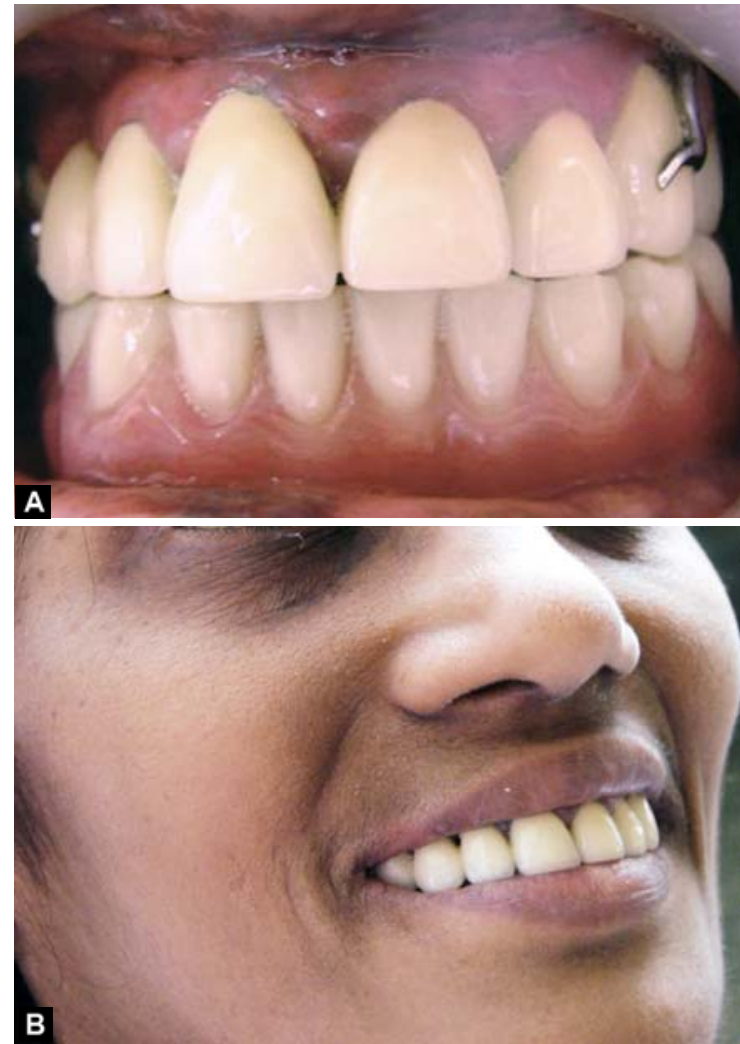

Figs 4A and B: (A) Postoperative, (B) postoperative profile view

Trismus is common when the masticatory muscles are bilaterally in the field of radiotherapy. Therefore, to prevent encroachment on the interocclusal distance for these patients, the vertical dimension of occlusion should be decreased 


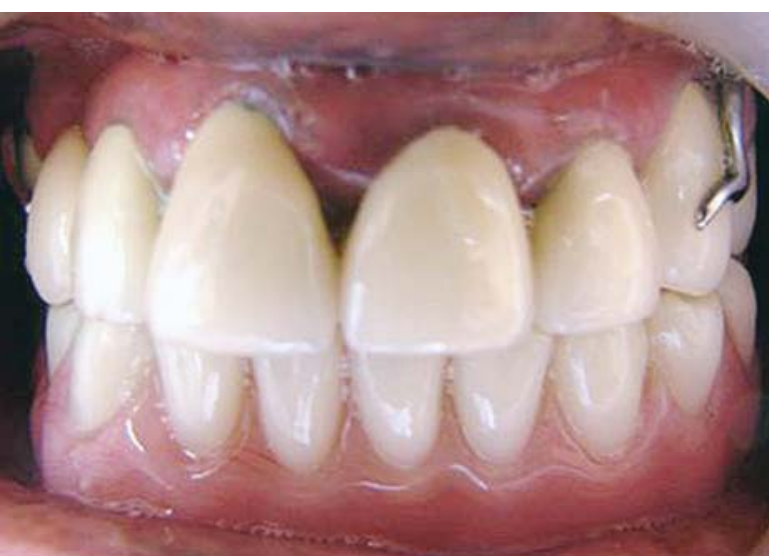

Fig. 5: Review after 9 months

accordingly. ${ }^{4}$ This aspect was kept in mind during the fabrication of both the overdentures and the cast partial denture. Flange extensions were thoroughly checked to prevent tissue ulcerations and the necessary guidelines were followed as far as the teeth selection and occlusion were concerned.

Postoperatively (Figs 4A and B) the patient was extremely comfortable and looked visibly pleased with the results of the treatment.

In addition, the patient has been on a continued maintenance regime, fluoride therapy and has been reviewed periodically (biweekly). Oral prophylaxis has been carried out every month and she has been advised to continue artificial saliva and maintain adequate oral hygiene. The patient has been followed up for 9 months since the conclusion of treatment and is asymptomatic since then (Fig. 5).

\section{DISCUSSION}

Prosthodontic therapy for xerostomia patients is challenging due to the limited choice of abutments, loss of vertical dimension, and poor occlusion. Often the patient, who is cured of cancer, faces an uphill struggle to return to some degree of normalcy. Sometimes, extreme adaptation is required to re-enter society successfully. At other times, the task proves too difficult and the patient fails in the attempt. Any technique or device that can help approximate a normal lifestyle will be of great benefit. ${ }^{16}$ Very few reports exist in literature with respect to oral rehabilitation of postirradiated cancer patients. ${ }^{3,4,6,13-15}$ The patient in question was a school teacher by profession and therefore, the ultimate challenge lay not just in complete oral rehabilitation of the patient but also enhancing her morale and confidence levels. Although the treatment plan devised for the patient was not unique in any way, the approach was evidence based and the onus was on achieving an overall remedy rather than attempting to try out something new and failing, causing further distress to an already demoralized patient. The fact that the patient has been well motivated to maintain excellent oral health all through her follow-up till 9 months further reiterates this viewpoint.

\section{SUMMARY AND CONCLUSION}

Xerostomic patients form a unique group of patients in whom prosthodontic treatment is challenging. Successful treatment depends on the knowledge and recognition of their particular problems and methods of prevention combined with skilful prosthodontics. Review and maintenance of any treatment is integral to its success. The present case of a successful rehabilitation of a patient with xerostomia and radiation caries following anticancer therapy, most definitely proves to be a stepping stone to success towards not only the oral but also the psychological well being of the patient.

\section{REFERENCES}

1. Kuntz R, Allen M, Osburn J. Xerostomia. Int J Pharm Compound 2000;4:1176-77.

2. Anon. Management of dry mouth and halitosis. Practitioner 1990;234:618-19.

3. Carl W. Dental management and prosthetic rehabilitation of patients with head and neck cancer. Head and Neck Surg 1980;3:27-42

4. Ladeane Fattore, Rosenstein Harry E, Louis Fine. Dental rehabilitation of the patient with severe caries after radiation therapy. Special Care in Dentistry 1986;6(6):258-61.

5. Cox FL. Endodontics and the irradiated patient. Oral Surg 1976;42:679-87.

6. Prosthodontic management of patients with xerostomia. Hong Kong Dental Journal 2005;2:132-34.

7. Kratochvil FJ. Partial removable prosthodontics. In: Dentistry. Philadelphia: Saunders Core Textbook 1988:11,27, 67,83-110, 132.

8. Phoenix Rodney D, Cagna David R, Defreest Charles F. In Stewart's Clinical Removable Partial Prosthodontics (4th ed). Quintessence Publishing Co Inc 2008:205-48.

9. Daher Tony, Hall Dan, Goodacre Charles J. Designing successful removable partial dentures. Compendium of Continuing Education in Dentistry, March 2006.

10. Benson D, Spolsky VW. A clinical evaluation of removable partial dentures with I-bar retainers (Part I). J Prosthet Dent 1979;41:246-54.

11. Stone E. Tripping action of bar clasps. J Am Dent Assoc 1936;23:596.

12. Kapur KK, Deupree R, Dent RJ, et al. A randomized clinical trial of two basic removable partial denture designs (Part I): Comparisons of five-year success rates and periodontal health. J Prosthet Dent 1994;72:268-82.

13. McDermott IG, Rosenberg SW. Overdentures for the irradiated patient. J Prosthet Dent 1984;51:314-17.

14. Rosenberg SW. Removable partial overdentures for the irradiated patient. Dent Clin North Am 1990;34:759-63.

15. Mirza FD, Dikshit JV. Use of implant prostheses following radiation therapy. J Prosthet Dent 1978;40(5):663-67.

16. Toljanic JA, Zucuskie TG. Use of a palatal reservoir in denture patients with xerostomia. J Prosthet Dent 1984;52:540-44. 